Conclusions: We isolated a subpopulation of more invasive glioblastoma cells selected in merosin substrate. Our findings suggest that merosin substrate is related to the brain invasion, and that increased expression of $\alpha_{1}, \alpha_{7}$ and $\beta_{1}$ integrin subunits, MMP2 activation, and fascin-actin interaction lead to increased motility and invasiveness in U87MG cell line.

\section{THE SIGNIFICANCES OF NUCLEAR AND CYTOPLASMIC OVEREXPRESSION OF METASTATIC TUMOR 1 IN HEPATOCELLULAR CARCINOMA}

Sang Hwa Lee, Hyun Hee Chu, Eunsil Yu

Department of Pathology, Asan Medical Center, University of Ulsan College of Medicine, Seoul, Korea

Background: Metastatic tumor antigen 1 (MTA1) protein is usually localized in the nuclei and its overexpression is suggested to be associated with vascular invasion, frequent postoperative recurrence, and poor survival in patients with hepatocellular carcinoma (HCC). Recently cytoplasmic MTA1 localization is recognized, however, its potential relationship with clinicopathological characteristics has not been examined. Aims: We investigated nuclear and cytoplasmic expressions of MTA1 to evaluate their relationship with clinicopathological characteristics in patients with HCC by immunohistochemistry. Methods: A total 263 patients with HCC who underwent resection from January 2013 to February 2015 were subjected. The patients previously treated by transarterial chemoembolization, radiofrequency ablation, and resection for recurrent HCC were excluded. Nuclear and cytoplasmic overexpressions of MTA1 were defined as positive in nucleus and cytoplasm more than $5 \%$ of tumor cells, respectively by immunohistochemistry.

Results: Nuclear overexpression was found in 154 patients. It was significantly associated with female gender $(p=0.039)$, viral etiology $(p=0.02)$, high Edmondson-Steiner grade ( $p=0.022$ in worst grade and $p=0.008$ in most grade), portal vein invasion $(p=0.017)$, Glisson capsule invasion $(p=0.026)$, high T stage $(p=0.016)$, and recurrence $(p=0.017)$. Interestingly, cytoplasmic overexpression was associated with beneficial factors for clinicopathological characteristics. Cytoplasmic overexpression was found in 183 patients. Negative for cytoplasmic overexpression was significantly associated with high histologic grade ( $p=0.003$ in most grade), portal vein invasion $(p=0.009)$, Glisson capsule invasion $(p=0.028)$, presence of satellite nodule $(p<0.001)$, high T stage $(p=0.003)$, recurrence $(p=0.02)$, and distant metastasis $(p=0.005)$. A total 109 cases with negative for nuclear overexpression, negative for cytoplasmic overexpression was significantly associated with high histologic grade $(p=0.01$ in most grade), Glisson capsule invasion ( $p=0.033)$, presence of satellite nodule $(p=0.012)$, high T stage $(p=0.033)$, and distant metastasis $(p=0.003)$.

Conclusion: Nuclear overexpression of MTA1 is associated with adverse clinicopathological factors, while cytoplasmic overexpression of MTA1 is associated with beneficial clinicopathological factors in HCC.

\section{CARBONIC ANHYDRASE 9 EXPRESSION IN PANCREATIC NEUROENDOCRINE TUMORS IS ASSOCIATED WITH AGGRESSIVE BEHAVIOR}

Sang Hwa Lee ${ }^{1}$, Joo Young $\mathrm{Kim}^{2}$, Soyeon $\mathrm{An}^{1}$,

Ki-Byung Song ${ }^{3}$, Dae Wook Hwang ${ }^{3}$, Song Cheol Kim ${ }^{3}$, Eunsil $\mathrm{Yu}^{1}$, Seung Mo Hong

${ }^{1}$ Department of Pathology, Asan Medical Center, University of Ulsan College of Medicine, ${ }^{2}$ Departmen of Pathology, Korea University, Anam Hospital, Seoul, and ${ }^{3}$ Department of Surgery, Asan Medical Center, University of Ulsan College of Medicine, Korea

Background: Pancreatic neuroendocrine tumors (PanNETs) are rare malignant endocrine tumors with diverse clinical behaviors. Therefore, identifying biomarkers is important for stratifying the clinical course and prognosis of PanNET patients. Carbonic anhydrase 9 (CA9) and vimentin are hypoxia and epithelialmesenchymal transition related protein, respectively. CA9 or vimentin expression was reported to have poor prognosis in many carcinomas. However, their expression has not been extensively evaluated with PanNET cases.

Aim: We aimed to investigate clinicopathologic correlations between CA9 and vimentin expression in PanNETs.

Methods: Immunohistochemical staining of CA9 and vimentin were assessed in 169 surgically resected PanNETs and compared with clinicopathologic factors, including patient survival.

Results: CA9 and vimentin expression was observed in 39 (23.1\%) and 37 (21.9\%) PanNET cases, respectively. PanNET patients with CA9 expression was associated with higher grade $(p<0.001)$, larger size $(p=0.002)$, higher $\mathrm{T}$ stage $(p<0.001)$, lymph node $(p=0.005)$ and distant $(p=0.026)$ metastasis, higher stage grouping $(p<0.001)$, lymphovascular $(p<0.001)$ and perineural $(p=0.004)$ invasions. Marginally significant survival difference was noted between CA9 positive and negative groups $(p=0.068)$. On the other hand, vimentin expression was significantly associated with CA9 expression $(p<0.001)$ although it does not associated with other clinicopathologic characteristics. Conclusion: CA9 expression is observed in subset of PanNETs, associated with advanced clinical stage, and has marginally worse survival time in PanNET patients.

\section{INDOLENT CD8-POSITIVE T-CELL LYMPHOMA OF THE GASTROINTESTINAL TRACT: A NOVEL ENTITY ANALOGOUS TO THE CUTANEOUS CD4-POSITIVE SMALL/MEDIUM T-CELL LYMPHOMA?}

$\underline{\text { Xiao-Qiu Li }}^{1,3}$, Wei-Ge Wang ${ }^{1,3}$, Ye Guo ${ }^{2,3}$, Xiao-Yan Zhou ${ }^{1,3}$, Xiao-Nan Hong ${ }^{2,3}$, Xiong-Zeng Zhu ${ }^{1,3}$

${ }^{1}$ Dept. of Pathology, ${ }^{2}$ Dept. of Medical Oncology, Fudan University Shanghai Cancer Center, and ${ }^{3}$ Dept. of Oncology, Shanghai Medical College, Fudan University, Shanghai, China

Background/Aim: Primary T- or NK (T/NK)-cell lymphomas of the gastrointestinal (GI) tract are uncommon diseases, comprising mainly some aggressive subtypes such as enteropathy-associated T-cell lymphoma and extranodal NK/T-cell lymphoma, nasal type. Rare cases of some T/NK-cell lymphoproliferative disorders with an indolent clinical course, however, 